\title{
Incidence of retinal changes in pregnant women due to pregnancy induced hypertension and its correlation with clinical profile
}

\author{
Himanshy Rai, Zakia Rahman*
}

Department of Obstetrics and Gynecology, Gajara Raja Medical College, Gwalior, Madhya Pradesh, India

Received: 04 February 2020

Accepted: 29 February 2020

\author{
*Correspondence: \\ Dr. Zakia Rahman, \\ E-mail: zakia.rahman08@gmail.com
}

Copyright: (C) the author(s), publisher and licensee Medip Academy. This is an open-access article distributed under the terms of the Creative Commons Attribution Non-Commercial License, which permits unrestricted non-commercial use, distribution, and reproduction in any medium, provided the original work is properly cited.

\begin{abstract}
Background: Pregnancy induced hypertension $(\mathrm{PIH})$ is associated with many other pathological complications. Pathological changes of this disease appear to be related to vascular endothelial dysfunction and its consequences. One such complication or effect of PIH is retinal changes.

Methods: This observational study was conducted of 110 cases over a period of 12 months from March 2018 to February 2019 in department of obstetrics of gynecology in Kamla Raja Hospital, G. R. Medical College, Gwalior, Madhya Pradesh. All the patients who fulfilled the diagnostic criteria of PIH admitted in the obstetric ward.

Results: Retinopathic changes were noted in $36.36 \%$ out of which grade I, grade II, grade III and grade IV have $11.82 \%, 8.18 \%, 10.19 \%$ and $5.45 \%$ cases respectively. In + proteinuria $10.9 \%$ of cases have positive findings, in ++ proteinuria $18.18 \%$ cases have positive findings, in $+++7.27 \%$ cases have retinopathy but severity increases with a grade of proteinuria. $40 \%$ cases retinopathic changes of mild preeclampsia have positive finding, $36.36 \%$ in severe preeclampsia and $23.64 \%$ in cases of eclampsia. As overall severity of PIH increases retinopathies in patients increases.

Conclusions: Visual symptoms are few in patients with PIH and often absent unless the macula is involved. Sudden onset of headache, which is resistant to routine therapy in these patients, may be the warning symptom before the onset of first convulsion. By repeated fundus examinations at regular intervals one can assess the severity of the disease and also response to treatment instituted.
\end{abstract}

Keywords: Pregnancy induced hypertension, Proteinuria, Retinopathic changes

\section{INTRODUCTION}

Pregnancy induced hypertension (PIH) is a hypertensive of pregnancy that occurs in absence of other cause of elevated blood pressure $140 / 90 \mathrm{mmHg}$ or a rise of 30 $\mathrm{mmHg}$ of systolic pressure or $15 \mathrm{mmHg}$ of diastolic pressure taken or two occasion after rest $4 \mathrm{hrs}$ apart in combination with generalized edema and/or proteinuria, after 20 weeks of pregnancy when there is significant proteinuria it is termed as preeclampsia (mild preeclampsia - blood pressure $>140 / 90$, proteinuria + and/or mild edema of legs, severe preeclampsia - blood pressure $>160 / 110$, proteinuria $++/+++$, or headache, cerebral or visual disturbance, impaired renal function test or rise serum creatinine). Seizures or coma as a consequence of PIH is termed as eclampsia. ${ }^{1-4}$ Pathological changes of this disease appear to be related to vascular endothelial dysfunction and its consequences (generalized vasospasm and capillary leak). ${ }^{5}$ The retinal vascular change generally but not always, correlate with severity of systemic hypertension. Vasospastic manifestations are reversible and retinal vessels rapidly return to normal after deliver. ${ }^{6}$ Retina is unique site where the vasculature in human body is visualized directly with help of ophthalmoscope. Eyes serve as a window through which vessels of body can be studied. Thus, a change in retinal arterioles may indicate a similar 
state of the placenta hence, it gives a reasonable idea of state of placental circulation and fetal well-being. ${ }^{7}$

\section{Classification $^{8}$}

Retinal changes (hypertensive retinopathy) were graded according to Keith Wagener classification

- Grade I - Mild generalized arteries attenuation particularly

- Grade II - More severe grade I + focal arteriolar attenuation

- Grade III - Grade II + hemorrhages hard exudates cotton wool spots

- Grade IV - Grade III + optic disc swelling (papilledema) or retinal detachment

Objective of this study was to determine the incidence of retinal changes in pregnant women due to pregnancy induced hypertension and its correlation with profile.

\section{METHODS}

This observational study was conducted of 110 cases over a period of 12 months from March 2018 to February 2019 in department of obstetrics of gynecology in Kamla Raja Hospital, G. R. Medical College, Gwalior, Madhya Pradesh. Written well informed and explained consent was taken from patient and attender in local language.

\section{Inclusion criteria}

- All the patients who fulfilled the diagnostic criteria of PIH admitted in the obstetric ward.

\section{Exclusion criteria}

- Patient who has preexisting diabetes or hypertension or renal disease placental abnormalities

- Hyperplacentosis: excessive expose to chorionic villi (molar, twin, diabetes in pregnancy)

- Pre-existing uteroplacental insufficiency

- Obesity BMI $>35 \mathrm{~kg} / \mathrm{m}^{2}$, insulin resistant preexisting vascular disease or thrombophilia's where excluded.

After taking history of any visual symptom's fundus examination was conducted bedside itself. Both pupils were dilated with $1 \%$ tropicamide eye drops and fundus examination was done by ophthalmologists with a direct ophthalmoscope in the ward. Hypertensive retinopathy changes seen on right and left both eyes were taken as positive finding in that patient. Age, direct or referred, GC, blood pressure, proteinuria, gravid, para, marital life, POG, family history, history of treatment was noted from the case record.

\section{Statistical analysis}

The results were analyzed using SPSS program Chi-square test was used to determine the association between the retinal change, blood pressure, proteinuria, gravid, severity, serum urea and serum creatinine and previous treatment of $\mathrm{PIH}$. A p value $<0.05$ was taken as significant.

\section{RESULTS}

Out of 110 patient, 58 patients were referred and 52 directly reported in department. The mean age of patients in study is 24.2 years (18-41 years). $63.63 \%$ of patients in study have no retinal changes.

Table 1: Distribution of cases according to age.

\begin{tabular}{|llllll|l|}
\hline Age (years) & Frequency (n) & No change & Grade I & Grade II & Grade III & Grade IV \\
\hline $18-21$ & 22 & 12 & 2 & 0 & 6 & 2 \\
\hline $22-25$ & 50 & 30 & 8 & 6 & 6 & 0 \\
\hline $26-29$ & 22 & 16 & 2 & 2 & 0 & 2 \\
\hline $30-33$ & 10 & 10 & 0 & 0 & 0 & 0 \\
\hline$>34$ & 6 & 2 & 1 & 1 & 0 & 2 \\
\hline
\end{tabular}

Table 2: Distribution of cases according to blood pressure.

\begin{tabular}{|c|c|c|c|c|c|}
\hline Blood pressure & No change & Grade I & Grade II & Grade III & Grade IV \\
\hline $140-150(\mathrm{~S})$ & 38 & 4 & 4 & 2 & 0 \\
\hline $90-100$ (D) & 46 & 8 & 4 & 2 & 4 \\
\hline $151-160(\mathrm{~S})$ & 26 & 8 & 2 & 6 & 2 \\
\hline $100-110(\mathrm{D})$ & 22 & 2 & 2 & 6 & 2 \\
\hline$>160(\mathrm{~S})$ & 6 & 2 & 3 & 4 & 4 \\
\hline$>110(\mathrm{D})$ & 2 & 3 & 3 & 4 & 2 \\
\hline
\end{tabular}

S: Systolic blood pressure, D: Diastolic blood pressure, $(p=0.001)$, statistically significant. 
Table 3: Distribution of cases according to proteinuria.

\begin{tabular}{|llll|ll|}
\hline Proteinuria & No change & Grade I & Grade II & Grade III & Grade IV \\
\hline Traces & 8 & 0 & 0 & 0 & 0 \\
\hline+ & 32 & 4 & 4 & 4 & 0 \\
\hline++ & 20 & 8 & 2 & 6 & 4 \\
\hline+++ & 10 & 1 & 3 & 2 & 2 \\
\hline$(\mathrm{p}=0.018$, statistically significant $)$. & & &
\end{tabular}

Table 4: Distribution of cases according to severity of disease.

\begin{tabular}{|llllll|}
\hline Severity of disease & No change & Grade I & Grade II & Grade III & Grade IV \\
\hline Mild preeclampsia & 34 & 6 & 2 & 2 & 0 \\
\hline Severe preeclampsia & 26 & 3 & 3 & 6 & 2 \\
\hline Eclampsia & 10 & 4 & 4 & 4 & 4 \\
\hline
\end{tabular}

$(\mathrm{p}=0.024$, statistically significant $)$.

Table 5: Distribution of cases according to parity.

\begin{tabular}{|llllll|}
\hline Parity & No change & Grade I & Grade II & Grade III & Grade IV \\
\hline Para 1 & 44 & 8 & 6 & 8 & 4 \\
\hline Para 2 & 16 & 4 & 3 & 2 & 2 \\
\hline Para $>2$ & 10 & 2 & 0 & 2 & 0 \\
\hline
\end{tabular}

$(\mathrm{p}=0.0034$, statistically significant $)$.

Table 6: Distribution of cases according to period of gestation.

\begin{tabular}{|c|c|c|c|c|c|}
\hline Period of gestation & No change & Grade I & Grade II & Grade III & Grade IV \\
\hline $20-28$ & 6 & 4 & 0 & 2 & 0 \\
\hline $29-34$ & 32 & 2 & 2 & 4 & 2 \\
\hline $35-40$ & 28 & 4 & 4 & 2 & 2 \\
\hline$>40$ & 4 & 3 & 3 & 4 & 2 \\
\hline
\end{tabular}

$(\mathrm{p}=0.041$, statistically significant $)$.

Retinopathic changes noted in $36.36 \%$ out of which grade I, grade II, grade III and grade IV have $11.82 \%, 8.18 \%$, $10.19 \%$ and $5.45 \%$ cases respectively Table 1 .

Table 2 shows that average of systolic blood pressure of all the findings $=156(140-220) \mathrm{mmHg}$ and average diastolic blood pressure $=124(90-130) \mathrm{mmHg}$. It can be concluded that as blood pressure increases, retinopathies increases Table 2 .

In + proteinuria $10.9 \%$ of cases have positive findings, in ++ proteinuria $18.18 \%$ cases have positive findings, in $+++7.27 \%$ cases have retinopathy but severity increases with a grade of proteinuria Table 3 . A total $40 \%$ cases retinopathic changes of mild preeclampsia have positive finding, $36.36 \%$ in severe preeclampsia and $23.64 \%$ in cases of eclampsia. As overall severity of PIH increases retinopathies in patients increases Table 4.

Positive retinal changes is diagnosed in $23.63 \%$ of para 1 patients $10 \%$ in para 2 patients and $2.73 \%$ in para $>2$ patients. Hereby it can be concluded that retinopathic changes are severe in para 1 patients Table 5. Average period of gestation $=34$ weeks 3 days $(20-40$ weeks gestation).

Positive retinal findings between gestational age of 20-28 weeks is $5.45 \%$, between $29-34$ weeks is $9.09 \%$, between $35-40$ weeks of gestation is $10.90 \%$ and $>40$ weeks of gestation is $10.90 \%$. Hereby, it can be concluded that severity of retinopathy increases as the gestational age increases Table 6.

Table 7: Distribution of cases according to history of previous treatment.

\begin{tabular}{|l|l|l|l|l|l|}
\hline History of previous treatment & No change & Grade I & Grade II & Grade III & Grade IV \\
\hline Present & 20 & 6 & 4 & 3 & 1 \\
\hline Absent & 50 & 7 & 5 & 9 & 5 \\
\hline
\end{tabular}

$(\mathrm{p}=0.24$, statistically insignificant $)$. 
Table 8: Distribution of cases according to biochemical markers.

\begin{tabular}{|l|ll|l|l|l|}
\hline Biochemical markers & No change & Grade I & Grade II & Grade III & Grade IV \\
\hline Serum urea & & & & & \\
\hline$\leq 4.5$ & 58 & 9 & 3 & 8 & 2 \\
\hline$>4.5$ & 12 & 4 & 6 & 4 & 4 \\
\hline Serum creatinine & & & & 6 & 2 \\
\hline$\leq 1 \mathrm{mg} / \mathrm{dl}$ & 50 & 12 & 5 & 6 & \\
\hline$>1 \mathrm{mg} / \mathrm{dl}$ & 20 & 1 & 4 & & \\
\hline
\end{tabular}

Patient with history of previous treatment significant changes on fundal examination was present in $12.72 \%$ of cases whereas with no history of treatment $23.63 \%$ changes were diagnosed Table 7.

Serum urea value $<4.5 \mathrm{mg} / \mathrm{dl}$, only $21.81 \%$ patients were diagnosed with retinopathy and $>4.5 \mathrm{mg} / \mathrm{dl}, 14.54 \%$ patients were diagnosed with retinopathy whereas serum creatinine $<1 \mathrm{mg} / \mathrm{dl}, 24.5 \%$ patients were diagnosed with retinopathy and in $>1 \mathrm{mg} / \mathrm{dl}, 11.81 \%$ patients were diagnosed with retinopathy Table 8 .

\section{DISCUSSION}

A total of 110 patients of PIH were examined. The mean of age 24.2 years (18-41 years).

The gestational period ranged from 20 weeks to 42 weeks, $70(63 \%)$ were the para $1.26(23.68 \%)$ were para 2 and $>$ para $2,14(12.72 \%)$ were multigravida.

Out of 110 patients, $40 \%$ mild preeclampsia, $36.36 \%$ had severe preeclampsia and $23.64 \%$ had eclampsia.

A total $40 \%$ patients had complaints of headache which was the most common symptoms while $25 \%$ patients had complained of blurred vision. Diplopia was seen in 1 patient and scotoma was seen in 1 patient.

Retinal changes, hypertensive retinopathy were noted in $36.36 \%$ patients. Grade I in $11.82 \%$, grade II $-8.18 \%$, grade III $-10.91 \%$ and grade IV $-5.45 \%$. Hemorrhage in 1 patient, exudates in 1 patients and retinal detachment were seen in 2 patients. There was statistically significant positive association of retinal changes and blood pressure $(\mathrm{p}=001)$. Proteinuria $(\mathrm{p}=0.018)$, severity of $\mathrm{PIH}(\mathrm{p}=$ $0.024)$, parity $(p=0.0034)$ and period of gestation with $p$ value $=0.041$ and in previous history of treatment with previous history of treatment $\mathrm{p}$ value $=0.24$ and biochemical markers $\mathrm{p}$ value $=0.31$ were not associated with retinopathy changes.

Pregnancy induced hypertension is responsible for maternal deaths, especially in the developing countries. In this study, the retinal changes were seen in $36.36 \%$ of preeclampsia/eclampsia. They found generalized arteriolar narrowing (11.81\%), cotton wool spot (8\%), hemorrhage (3.6\%) and serous retinal detachment (1.8\%). They noted the resolution of all the above retinal changes except narrowing of arteries during the puerperium period.

The visual system may be affected in $30 \%$ to $100 \%$ of patients with PIH; the most common abnormality seen in the fundus is narrowing of retinal arterioles. ${ }^{2}$ Various changes in the fundus and visual problems reported in patients of preeclampsia and eclampsia include spasm and focal/generalized narrowing of retinal arterioles, hemorrhages, exudates, peripapillary or focal retinal edema, serous retinal detachment, isolated cases of acute ischemic optic neuropathy, transient blindness, cortical blindness, bilateral retinal detachment, exudative retinal detachment in one eye and severe macular edema in the other eye, retinal pigment epithelial lesions, temporary decrease in vision secondary to severe retinal arteriolar spasm and retinal edema, permanent blindness secondary to central retinal artery occlusion and optic atrophy. Although transient blindness has been reported in $1 \%$ to $3 \%$ of patients with eclampsia, with current methods of treatment the present incidence is probably much lower. Optic atrophy secondary to retinal vascular involvement is unusual but may cause visual impairment. ${ }^{9,10}$

Jaffe and Schatz from USA have reported significant correlation between the reduction in arteriole to vein ratio, number of focal arteriolar constrictions and severity of preeclampsia. ${ }^{11}$ They did not find any hemorrhages, exudates, cotton wool spots, or retinal detachment in their study of 17 mild preeclamptic and 14 severe preeclamptic patients.

In a study of 275 cases of preeclampsia and 125 cases of eclampsia, Reddy from India has reported retinal changes in $53.4 \%$ preeclampsia and in $71.2 \%$ in eclampsia patients (overall 59\%, 236 out of 400). ${ }^{12}$ The most common retinal change noted was narrowing of arterioles $(45.7 \%, 183$ out of 400 cases). He found that retinal changes were significantly more in patients with severe hypertension.

Tadin et al, from Croatia have reported $45 \%$ of retinal changes in their study of 40 patients with PIH. ${ }^{13}$ They found a statistical correlation between proteinuria, blood pressure and hypertensive retinopathy. The degree of retinopathy was directly proportional to severity of 
preeclampsia. They stated that hypertensive retinopathy is a valid and reliable prognostic factor in determining the severity of preeclampsia; examination of fundus is a valuable and necessary diagnostic procedure in pregnant women with preeclampsia.

Karki et al from Nepal have reported $13.7 \%$ of fundus changes in their study of 153 subjects with PIH. ${ }^{14}$ They assessed the foetal outcome in these patients and concluded that retinal and optic nerve head changes were associated with low birth weight; choroidal and optic nerve head changes were associated with low Apgar score; and fundus evaluation in patients with $\mathrm{PIH}$ is an important procedure to predict adverse foetal outcomes.

The prevalence of hypertensive retinopathy changes $(59 \%)$ seen in this study is higher than $13.7 \%, 21.5 \%$, $45 \%$, but similar to $59 \%$ reported in the literature. The absence of hemorrhages and exudates observed in present study has been supported by Jaffe and Schatz. ${ }^{11}$

Exudative retinal detachment is seen rarely in $\mathrm{PIH}$ patients. It is thought to be caused by choroidal ischemia. Retinal pigment epithelial lesions, called Elscchnig spots, may also be found in preeclamptic patient with choroidal infarcts. The prognosis in these cases is good, with visual symptoms and retinal pigment epithelial changes resolve spontaneously within weeks of delivery. ${ }^{15}$ Presence of macular edema or papilledema or retinal detachment are the warning signs for termination of pregnancy to save the vision of the mother. The management of retinal detachment is not surgery, but termination of pregnancy after controlling blood pressure so that vision can be saved in the affected eye.

Cortical blindness refers to reduced vision from bilateral damage to any portion of the visual pathway's posterior to the lateral geniculate nucleus. Eye examination is typically normal, including a normal pupillary light reflex. It can occur in ante partum and postpartum period, lasting for several hours to several days in preeclampsia and eclampsia patients. ${ }^{16}$ Other presenting symptoms include headache, seizures and loss of consciousness. MRI shows hypertense signals on T2-weighted images and hypotense signals on T1-weighted images in occipital cortex. These findings are consistent with transient ischemic events as a result of cerebral edema. Management includes magnesium sulphate for seizure prophylaxis, anti-hypertensives for severe hypertension, fluid restriction to avoid worsening of cerebral edema, ophthalmologic and neurologic consultation as well as neuroimaging. Prompt delivery is curative, with resolution of neuroimaging findings. ${ }^{17}$

The retinal changes were more often seen in patients with severe hypertension, severe proteinuria and severity of the disease in present study; they were significantly associated with these factors lessely related to serum urea and creatinine values that are biochemical markers of PIH. A similar association between hypertensive retinopathy and the above three parameters was reported in earlier studies. This study did not find any case of serous retinal detachment in present study which is similar to the previously reported studies. However, Rasdi et al reported a case of serous retinal detachment from Malaysia. ${ }^{7}$

In general, it is believed that the presence of changes in the retinal arterioles and retinal hemorrhages may indicate similar changes in the placenta. Since the wellbeing of the foetus depends on the placental circulation, ophthalmoscopic examination of mother's fundus may give a clue to similar micro-circulation changes in the placenta and indirectly to the foetal wellbeing. Fundus examination in patients with $\mathrm{PIH}$ is an important clinical evaluation to predict adverse foetaloutcomes. ${ }^{14}$

\section{CONCLUSION}

Visual symptoms are few in patients with PIH and often absent unless the macula is involved. Sudden onset of headache, which is resistant to routine therapy in these patients, may be the warning symptom before the onset of first convulsion. The presence of multiple hard exudates in retina may indicate albuminuria retinopathy and the possibility of damage to the kidneys. The presence of papilledema in the eyes may indicate raised intracranial tension and such patients may develop convulsions. In cases of toxaemia of pregnancy, the retinal changes usually regress with decrease in blood pressure and may disappear completely after delivery due to lack of placental toxins. Therefore, by repeated fundus examinations at regular intervals one can assess the severity of the disease and also response to treatment instituted.

Funding: No funding sources Conflict of interest: None declared

Ethical approval: The study was approved by the Institutional Ethics Committee

\section{REFERENCES}

1. Reddy SC, Nalliah S, George SRA, Who TS. Fundus changes in pregnancy induced hypertension. Int $\mathrm{J}$ Ophthalmol. 2012;5(6):694-7.

2. Richard RO. Pregnancy induced hypertension (preeclampsia-ecclampsia) In: Schachat AP, Murphy RB, editors. Retina. $2^{\text {nd }}$ ed. St Louis: Mosby; 1994:1405-12.

3. Nalliah S, Abdullah AR. Eclampsia in Kelantan. Med J Malaysia. 1990;45(1):49-56.

4. Noraihan MN, Sharda P, Jammal AB. Report of 50 cases of eclampsia. J Obstet Gynaecol Res. 2005;31(4):302-9.

5. Nalliah S, Thavarasha AS. Transient blindness in pregnancy induced hypertension. Int $\mathrm{J}$ Gynaecol Obstet. 1989;29(3):249-51. 
6. Achanna S, Monga D, Sivagnanam. Transient blindness in pregnancy induced hypertension. Asia Oceania J ObstetGynaecol. 1994;20(1):49-52.

7. Rasdi AR, Nik-Ahmad-Zuki NL, Bakiah S, Shatriah I. Hypertensive retinopathy and visual outcome in hypertensive disorders in pregnancy. Med $\mathrm{J}$ Malaysia. 2011;66(1):42-7.

8. Kanski JJ. $2^{\text {nd }}$ ed. Oxford: Butterworth Heinmann. Clinical ophthalmology: a systematic approach; 1989;329.

9. Gandhi J, Ghosh S, Pillari VT. Blindness and retinal changes in preeclamptic toxemia. Pascal Franc Bibliog Databases. 1978;78(12):1930-2.

10. Mathew M, Rajan CK, Gulati N. Amaurosis in toxemia of pregnancy. Indian $\mathrm{J}$ Ophthal. 1975;23(1):25-6.

11. Jaffe G, Schatz H. ocular manifestations of preeclampsia. Am J Ophthal. 1987;103(3Pt1):30915.

12. Reddy SC. Ocular fundus changes in toxemia of pregnancy. The Antisept. 1989;86(7):367-72.

13. Tadin I, Bojić L, Mimica M, Karelović D, Dogas Z. Hypertensive retinopathy and preeclampsia. Coll Antropol. 2001;25(Suppl 0):77-81.
14. Karki P, MallaKP, Das H, Uprety DK. Association between pregnancy induced hypertensive fundus changes and fetal outcome. Nepal J Ophthalmol. 2010;2(1):26-30.

15. Saito Y, Tano Y. Retinal pigment epithelial lesions associated with choroidal ischemia in preeclampsia. Retina. 1998;18(2):103-8.

16. Cunningham FG, Fernandez CO, Hernandez C. Blindness associated with preeclampsia and eclampsia. Am J Obstet Gynecol. 1995;172(4Pt1):1291-8.

17. Apollon KM, Robinson JN, Schwartz RB, Norwitz ER. Cortical blindness in severe preeclampsia: computed tomography, magnetic resonance imaging, and single-photon-emission tomography findings. Obstet Gynaecol. 2000;95(6Pt2):1017-9.

Cite this article as: Rai $\mathrm{H}$, Rahman $\mathrm{Z}$. Incidence of retinal changes in pregnant women due to pregnancy induced hypertension and its correlation with clinical profile. Int J Reprod Contracept Obstet Gynecol 2020;9:1640-5. 